\title{
Alireza Khosrowzadeh, Aliashgar Norouzi, Rika Gyselen, Hossein Habibi. "Administrative Seal Impressions on Bullae Discovered on Tappe Bardnakoon"
}

Rémy Boucharlat

\section{(2) OpenEdition}

Journals

Édition électronique

URL : https://journals.openedition.org/abstractairanica/53372

DOI : 10.4000/abstractairanica.53372

ISSN : 1961-960X

Éditeur :

CNRS (UMR 7528 Mondes iraniens et indiens), Éditions de l'IFRI

Référence électronique

Rémy Boucharlat, « Alireza Khosrowzadeh, Aliashgar Norouzi, Rika Gyselen, Hossein Habibi.

"Administrative Seal Impressions on Bullae Discovered on Tappe Bardnakoon" », Abstracta Iranica [En ligne], Volume 42-43 | 2021, document 65, mis en ligne le 30 décembre 2021, consulté le 13 décembre 2022. URL : http://journals.openedition.org/abstractairanica/53372 ; DOI : https://doi.org/10.4000/ abstractairanica.53372

Ce document a été généré automatiquement le 13 décembre 2022.

Tous droits réservés 


\title{
Alireza Khosrowzadeh, Aliashgar Norouzi, Rika Gyselen, Hossein Habibi. "Administrative Seal Impressions on Bullae Discovered on Tappe Bardnakoon"
}

\author{
Rémy Boucharlat
}

\section{RÉFÉRENCE}

Alireza Khosrowzadeh, Aliashgar Norouzi, Rika Gyselen, Hossein Habibi.

"Administrative Seal Impressions on Bullae Discovered on Tappe Bardnakoon" in R. Gyselen (ed.). Persia (522 BCE - 758 CE). Primary Sources, Old and New. Res Orientales XXVIII, Bures-sur Yvette : Groupe pour l'Étude de la Civilisation du Moyen-Orient, 2020, p. 83-112.

1 Cet article est concentré sur les sceaux administratifs sassanides, dont R. Gyselen est la spécialiste incontestée. Ils ont été découverts lors des deux campagnes de fouilles en 2017 et 2018 à Tappeh Bardnakon, abrégé TB. Chacun d'eux, lorsqu'il est bien conservé, porte le nom de l'administration territoriale et celui du lieu d'où est exercée l'autorité. Le corpus est de 22 empreintes mais quelques-unes reproduisent le même sceau (voir en Annexe p. 105-111 le précieux corpus complet des empreintes de sceaux avec description complète, dessin et photo).

2 La contribution de l'archéologie à la géographie historique sassanide est la provenance depuis un site bien localisé, ce qui en fait la troisième collection de ce type dans le sud de l'Iran avec Qasr-i Abu Nasr, près de Shiraz et Tol-e Qaleh Seyfabad (non publiée) dans la région de Bishapur. Ces sceaux ne représentent que $4 \%$ de l'ensemble des bulles 
découvertes à $\mathrm{TB}$, mais on ne sait pas quelle est l'importance du pillage qui a précédé la fouille.

3 À la différence des territoires mentionnés dans ces deux autres archives, qui sont assez peu nombreux et le plus souvent voisins du site, ceux des sceaux de TB couvrent une plus vaste aire géographique, couvrant plusieurs régions (au sens administratif du terme à l'époque sassanide, chacune comprenant plusieurs cantons administré par un mowūh, juridiction religieuse et judiciaire) : Spahān (Gay) principalement, représentée par six empreintes du mowūh de Rāwar-kust-ī-rōdbār , probablement la région dans laquelle TB était située, Hūzestān (Rām-Ohrmazd, Ohrmazd-Ardašir and Weh-AndiyokŠābuhr) and Pārs (Ig).

4 Cette collection est une importante contribution à la géographie historique sassanide et elle semble datée, comme les deux autres, des $\mathrm{VI}^{\mathrm{e}}$ et $\mathrm{VII}{ }^{\mathrm{e}}$ siècle. La paléographie n'est d'aucun secours, car les inscriptions de ce même corpus attestent de différences paléographiques et de graphies, voire des erreurs.

\section{AUTEURS}

\section{RÉMY BOUCHARLAT}

UMR 5133 CNRS-Université de Lyon 